\title{
A LOCALLY COMPACT SEPARABLE METRIC SPACE IS ALMOST INVARIANT UNDER A CLOSED MAPPING
}

\author{
BY EDWIN DUDA
}

Communicated by P. E. Conner, October 25, 1963

For a given mapping (continuous transformation) $f$ of a topological space $X$ onto a topological space $Y$ it has always been of interest to determine what properties of $X$ carry over to $Y$. Under the hypothesis that $f$ is a closed mapping it is known that normality [1], and paracompactness [5] are invariants. If $X$ is metric and $f$ is closed then as a consequence of results of Vainnšteĭn [2], Whyburn [1], and Stone [4], it is known that $Y$ is weakly separable if and only if each point inverse has a compact frontier. From [1] we obtain the result that if $X$ is perfectly separable, $f$ is closed and $Y$ is weakly separable then $Y$ is a separable metric space. Let $f$ be a closed mapping of a locally compact separable metric space $X$ onto a topological space $Y$, or, equivalently, let $G$ be an upper semi-continuous decomposition of $X$ into closed sets. We say a set $S$ is a scattered set if every subset of $S$ is closed. Our results show that $Y$ (or the decomposition space $M$ ) minus a scattered set $S$, which has at most a countable number of points, is also a locally compact separable metric space. The techniques of proof are standard and will not be included here.

THEOREM 1. Let $G$ be an upper semi-continuous decomposition of a locally compact separable metric space into closed sets. Let $F$ be the union of the noncompact elements of $G, M$ the decomposition space determined by $G$, and $\phi$ the natural mapping of $X$ onto $M$. The following are valid.

(i) $F$ is a closed set.

(ii) For an arbitrary compact set $K$ only a finite number of elements of $G$ in $F$ can intersect $K$.

(iii) $F$ contains at most countably many elements of $G$.

(iv) The union of any subcollection of elements of $G$ in $F$ is a closed set.

(v) $M$ is weakly separable at $y$ if and only if the frontier of $\phi^{-1}(y)$ is compact (Stone [4]).

(vi) If $\left\{g_{n}\right\}$ is a convergent sequence of compact elements of $G$ with a nonempty limiting set $h$, then the set $K=\bigcup g_{n} \cup h$ is compact.

Let $F^{\prime}$ be the subset of $F$ composed of the union of the elements 
that do not have a compact frontier. The set $F^{\prime}$ is closed by (iv) and $S=\phi\left(F^{\prime}\right)$ is closed and is at most a countable set of points. Using this notation we have:

TheOREM 2. The set $M-S$ is a locally compact separable metric space, where $S$ is a scattered set having at most a countable number of points.

A lower semi-continuous decomposition of a metric space $X$ has the property that if $\left\{g_{n}\right\}$ is a converging sequence of elements of $G$ with a nonempty limiting set $h$ then $h$ is an element of $G$. For an open mapping replace the $g_{n}$ by point inverses. By (vi) of Theorem 1 it is easy to see how to obtain the following theorem which is given in a slightly stronger form by Wallace [3] and Whyburn [6].

Theorem 3. If $G$ is both an upper semi-continuous and a lower semicontinuous decomposition of a locally compact connected separable metric space $X$, then all the elements of $G$ are compact.

To see that $S$ can be infinite consider the decomposition of the plane into the vertical lines whose equations are $x=n, n$ an integer, and the individual points not on these lines.

Added in proof. Separability can be omitted in the hypotheses provided that the countability of $F$ and $S$ is excluded from the conclusions.

\section{BIBLIOGRAPHY}

1. G. T. Whyburn, Open and closed mappings, Duke Math. J. 17 (1950), 69-74.

2. I. A. VaǏnšteY̌n, On closed mappings of metric spaces, Dokl. Akad. Nauk SSSR 57 (1947), 319-321. (Russian)

3. A. D. Wallace, Some characterizations of interior transformations, Amer. J. Math. 61 (1939), 757-763.

4. A. H. Stone, Metrizability of decomposition spaces, Proc. Amer. Math. Soc. 7 (1956), 690-700.

5. E. Michael, Another note on paracompact spaces, Proc. Amer. Math. Soc. 8 (1957), 822-828. 226.

6. G. T. Whyburn, Continuous decompositions, Amer. J. Math. 71 (1949), 218-

UNIVERSITY OF MIAMI 